\title{
The Memory Aid study: protocol for a randomized controlled clinical trial evaluating the effect of computer-based working memory training in elderly patients with mild cognitive impairment (MCl)
}

\author{
Marianne M Flak ${ }^{1,2^{*}}$, Susanne S Hernes ${ }^{1}$, Jon Skranes ${ }^{2,3}$ and Gro CC Løhaugen ${ }^{2,3}$
}

\begin{abstract}
Background: Mild cognitive impairment $(\mathrm{MCl})$ is a condition characterized by memory problems that are more severe than the normal cognitive changes due to aging, but less severe than dementia. Reduced working memory (WM) is regarded as one of the core symptoms of an $\mathrm{MCl}$ condition. Recent studies have indicated that WM can be improved through computer-based training. The objective of this study is to evaluate if WM training is effective in improving cognitive function in elderly patients with $\mathrm{MCl}$, and if cognitive training induces structural changes in the white and gray matter of the brain, as assessed by structural MRI.

Methods/Designs: The proposed study is a blinded, randomized, controlled trail that will include 90 elderly patients diagnosed with $\mathrm{MCl}$ at a hospital-based memory clinic. The participants will be randomized to either a training program or a placebo version of the program. The intervention is computerized WM training performed for 45 minutes of 25 sessions over 5 weeks. The placebo version is identical in duration but is non-adaptive in the difficulty level of the tasks. Neuropsychological assessment and structural MRI will be performed before and 1 month after training, and at a 5-month follow-up.

Discussion: If computer-based training results in positive changes to memory functions in patients with $\mathrm{MCl}$ this may represent a new, cost-effective treatment for $\mathrm{MCl}$. Secondly, evaluation of any training-induced structural changes to gray or white matter will improve the current understanding of the mechanisms behind effective cognitive interventions in patients with $\mathrm{MCl}$.
\end{abstract}

Trial registration: ClinicalTrials.gov NCT01991405. November 18, 2013.

\section{Background}

Mild cognitive impairment (MCI) is a clinical condition characterized of a reduction in memory and/or other cognitive processes that are insufficiently severe to be diagnosed as dementia, but are more pronounced than the cognitive decline associated with normal aging. In Norway, MCI and dementia represent major health concerns, which will be even more pronounced in the

\footnotetext{
* Correspondence: marianne.moretro.flak@sshf.no

'Department of Medicine, Geriatric Unity, The Memory Clinic, Sørlandet Hospital, Arendal, Norway

${ }^{2}$ Department of Pediatrics, Sørlandet Hospital, Arendal, Norway

Full list of author information is available at the end of the article
}

future because of the demographic shift that is resulting in an increased number of elderly citizens. The economic burden of these disorders both for the individual and for society is a heavy one $[1,2]$. Currently, no treatment is available for enhancing memory function or preventing further decline in patients with MCI. Impairment of memory and learning, including the working memory (WM) capacity, characterizes an MCI condition [3].

WM is a theoretical construct referring to the structures and processes associated with our ability to keep information in mind, while we actively use and respond to the information $[4,5]$. Individuals with WM deficits have trouble 
remembering information even for a few seconds, find it difficult to keep track of what they are doing as they work, or forget what they are supposed to retrieve when sent on an errand [6]. Previously, a person's WM capacity was presumed to be constant and unmodifiable by stimulation or other external factors. Recently, the 'neuroplasticity paradigm' has gained support, and a growing body of research has focused on the brain's modification capabilities. The notion that mentally stimulating activities may affect cognitive processes, including memory, has also been linked to a 'use it or lose it' hypothesis [7].

Different interventions have been developed to try to enhance or preserve cognitive function [8]. Strategic interventions (by which an individual learns strategies to circumvent and compensate for the cognitive deficits) may be difficult for patients with MCI patients because of their memory problems. We use the definition suggested by Jolles and Crone [9] of cognitive training as the process of improving cognitive functioning by means of practice and/or intentional instruction. The intervention method in this study can be classified as a process-based approach, which involves repeated performance (that is, practice) of demanding (WM) tasks, for which an underlying change in neural correlates is hypothesized $[9,10]$. WM capacity is associated with the organization of the white matter tracts that constitute the network between the parietal and the frontal lobes [11]. This assumption has been supported by the finding that adaptive WM training causes structural changes of the nerve tracts in the brain [12-14]. It is plausible to expect that performance gain in WM would improve various everyday activities that place high demands on WM capacity [9,15-17].

Positive effects of WM training have been reported in diverse clinical groups, including children with attention deficit hyperactivity disorder (AD/HD), as well as preschool children and youths born preterm with very low birth weight [18-32]. Reviews have reported moderate to large beneficial effects of cognitive exercises on memoryrelated outcomes $[8,10,33]$. Enhancing the function in specific domains by training the impaired skill(s) demonstrate the greatest overall effect on functioning compared with non-training methods such as memory strategies and compensatory strategies in patients with Alzheimer's disease [8]. Several studies have reported improved performance on tasks included in the intervention (trained tasks) as well as improvement on non-trained WM tasks and other cognitive tasks that require spatial skills and involve complex problem-solving skills. However, there is a lack of studies on effective training interventions for $\mathrm{MCI}$ patients, and the few randomized controlled trials (RCTs) that exist have shown divergent results $[16,33,34]$. In addition, the existing studies have the limitations of non-standardized intervention methods and low statistical power, rendering conclusions on these matters challenging. It is therefore of great importance to perform RCTs investigating the effects of specific cognitive interventions in elderly patients with MCI who are at high risk of developing dementia. In addition, the proposed study seeks to investigate individual differences in training outcome and its relation to dementia biomarkers such as tau, phospho-tau, and $\beta$-amyloid proteins as well as candidate genes such as $A P O E$ and $L M X 1 A$. A recent study by Bellander et al. [35] indicates that certain combinations of the different alleles of the $L M X 1 A$ gene might predict those healthy adults most likely to benefit from WM training. This gene is involved in both development and maintenance of dopamineproducing neurons, which are related to WM function [36-40]. If this applies to the MCI group, such a biomarker could be a marker for the effects of the intervention and thereby correlate with the feasibility of this particular intervention. This article describes a study that it aimed to increase knowledge of the neuropsychological status in patients with MCI, as well as the feasibility and possible effects of a cognitive training intervention.

\section{Methods/Design \\ Objectives}

The primary objective of this blinded RCT is to evaluate the hypothesis that standardized computer-based WM training is effective in improving WM capacity over time in patients diagnosed with MCI. Second, we want to assess whether computer-based adaptive WM training in patients with MCI has transfer effects to other cognitive functions, including memory, attention, and executive functions, as well as activities of daily living, measured by a battery of neuropsychological tests. Third, any effects of WM training on the structural changes in white and gray matter of the brain will be investigated, measured by multimodal magnetic resonance imaging. Finally, possible genetic biomarkers ( $L M X 1 A$ and APOE genotypes) related to a positive effect or lack of effect of the training will be examined.

Study approval was given by the Norwegian regional committee for medical and health research ethics, southeastern region (reference number 2013/410/REK Sør-øst). The study will be reported in accordance with both the CONSORT statement and the CONSORT statement for non-pharmacological interventions $[41,42]$.

\section{Participants}

In total, 90 patients diagnosed with MCI recruited from memory clinics at Sørlandet Hospital, Arendal, The Hospital of Telemark, and Oslo University Hospital will be included in the study. The patients will undergo standard medical examination and neuropsychological testing to ensure correct diagnosis of MCI. Informed consent will be obtained from each participant. 


\section{Inclusion and exclusion criteria}

Patients who meet the Peterson diagnostic criteria of MCI [2]: 1) memory problems, preferably confirmed by an informant, 2) memory impairment in accordance with age and education, 3) preserved general cognitive function, 4) intact activities of daily living, and (5) absence of dementia (in accordance with the ICD-10/DMS-IV criteria). Patients who have had head trauma with post-traumatic loss of consciousness for at least 30 minutes during their lifespan, or who have loss of senses (blindness, deafness) or photosensitive epilepsy or who are unsuitable for magnetic resonance imaging because of implanted metal foreign bodies or severe claustrophobia will be excluded from the study.

\section{Outcome measures and assessment procedures}

Baseline assessment will be completed prior to randomization and the first intervention session.

\section{Neuropsychological assessment}

The neuropsychological/cognitive evaluation includes the administration of several commonly used measures (tests, interview, and questionnaires) of cognitive abilities and daily functions. Measures of the patient's cognitive status (intelligence, memory, processing speed, attention, and executive functions) will include a wide range of standardized neuropsychological tests, as well as tests assessing prefrontal functions, as these may mediate WM decline [43]. Measures are administered as a combination of paper-andpencil tests and a computer-administered task. The sensitivity and specificity of the assessment methods have been described previously $[44,45]$. To correct for learning effects on repeated tests, alternative test versions or forms will be used when possible. The selected neuropsychological tests and assessed functions is described in Table 1.

\section{Interview and questionnaires}

The following interviews and questionnaires will be used.

- Vineland Adaptive Behavior Scales, second edition (interview). This structured interview measures

Table 1 Summary of assessed cognitive functions and tests

\begin{tabular}{ll}
\hline Function: & Tests: \\
\hline $\begin{array}{l}\text { General cognitive ability (IQ) } \\
\text { Auditory and visual WM }\end{array}$ & WAIS-IV \\
$\begin{array}{l}\text { Digit Span, Spatial Span/Span Board } \\
\text { and Letter-Number Sequencing, PASAT } \\
\begin{array}{l}\text { Verbal and visual learning, } \\
\text { immediate and delayed } \\
\text { memory }\end{array}\end{array}$ & WMS-III, CVLT-II \\
$\begin{array}{l}\text { Attention and executive } \\
\text { functions }\end{array}$ & $\begin{array}{l}\text { D-KEFS subtests including: Trails 1 to 5, } \\
\text { Color Word Interference, Tower, Verbal } \\
\text { fluency, Design fluency. Supplemental } \\
\text { test: lowa Gambling Task }\end{array}$ \\
\hline
\end{tabular}

adaptive abilities in three broad domains: communication, activities of daily living, and social skills.

- Behavior Rating Inventory of Executive Functions Adult version (BRIEF-A). This questionnaire measures nine domains of executive functions (inhibition, shifting, emotional control, self monitoring, initiating, WM, planning/organizing, task monitoring, and organization of materials) through a self-reported and an informant version.

- Behavioral assessment of the Dysexecutive Syndrome Questionnaire (DEX). Assesses problems in executive function through a self-report and an informant version.

\section{Magnetic resonance brain imaging}

The patients will undergo brain MRI scanning at baseline and at 1 and 4 months post-training, using an optimized protocol. Following a pilot scan, a three-dimensional (3D) magnetization-prepared rapid gradient echo (MP-RAGE) scan will be performed (sagittal, echo time 3.47, repetition time 2400, TI 1000, flip angle (FA) 8 degrees, $1.2 \mathrm{~mm}$ resolution covering the whole brain).

Diffusion tensor imaging (DTI) scan will be performed (axial (non-oblique), 68 slices, field of view $=240 \mathrm{~mm}$, $2.5 \mathrm{~mm}$ slices without gap; $2.5 \times 2.5 \mathrm{~mm}$ in-plane resolution; repetition time9.500, echo time 91, two averages, diffusion $(b=0.1000), 30$ diffusion directions. In addition a 3D-T2 space dark fluid (FLAIR) image (sagittal, echo time 335 , repetition time 5000, TI 1800, turbo factor 242, $1.2 \mathrm{~mm}$ resolution covering the whole brain) and a T2* image (axial TE 25, TR 830, flip angle 20 degrees) will also be acquired during the initial scan for clinical evaluation to exclude microbleeds. Total scanning time for the initial scan will be close to 30 minutes. The follow-up scans will be under 25 minutes. All scans will be reviewed qualitatively by a radiologist to screen for possible brain lesions or structural abnormalities. By using tract-based spatial statistics (TBSS), the radiologist will measure fractional anisotropy, and mean diffusivity values in central white matter tracts before and after training. Automated morphometry to evaluate cortical thickness, surface area, and total gray and white matter volumes, as well as deep nuclei volumes will be performed.

\section{Biological/biochemical samples, study biobank, and DNA collection}

One oral cotton swab will be collected for genotype analyses for $A P O E$ and $L M X 1 A$ alleles. DNA will be extracted from saliva collected using an Oragene Self collection Kit (DAN Genoteck, Inc. Ottawa, Ontario, Canada). Genomic DNA will be subjected to Restriction Fragment Length Polymorphism analyses (RFLP-PCR). Approximately $3 \mathrm{ng}$ of genomic DNA for $L M X 1 A$ will 
be amplified by PCR using the primer LMX-5': $5^{\prime}$ CTCGCCTCCAGGAA TGGGTGTTGTA-3' and LMX3': 5'-GCCACGAGGAACTTGTGAGAGGGTT-3' with the following conditions: denaturation at $94^{\circ} \mathrm{C}$ for 5 minutes, followed by 25 cycles at $94^{\circ} \mathrm{C}$ for 30 seconds, annealing at $64^{\circ} \mathrm{C}$ for 30 seconds and extending at $72^{\circ} \mathrm{C}$ for 30 seconds. Then $15 \mu \mathrm{l}$ of the amplification PCR products will be digested directly by $2.5 \mathrm{U}$ of the restriction enzyme $M s l \mathrm{I}$ (R0571S, New England Biolabs, Beverly, MA, USA) for 2 hours at $37^{\circ} \mathrm{C}$. The digested PCR products will then be analyzed on $4 \%$ agarose gel, and visualized using GelGreen ${ }^{\text {тм }}$ Nucleic Acid Gel Stain (89139-144, Biotium, Hayward, CA, USA).

\section{Primary outcome measure}

Primary outcome measure is the spatial span board task from the Wechsler Memory Scale, third edition (WMS-III).

\section{Secondary outcome measures}

Secondary outcome measureszare the structural MRI changes, the results on neuropsychological test measuring verbal and visual learning and memory, and the response pattern on the questionnaires.

\section{Intervention}

The intervention method is classified as an adaptive, intensive, and standardized computerized WM training program $\left(\right.$ Cogmed $\left.^{\circledR}\right)$, developed by Professor T. Klingberg at the Karolinska Institute, Stockholm, Sweden $[29,46]$. Both the adaptive and the 'placebo' training groups will use the program approximately 30 to 40 minutes a day, 5 days a week for 5 weeks. The training is considered 'adaptive', which means that the difficulty level of the tasks increases during the sessions according to the individual level of mastering for each participant, making the patient work at their maximum capacity at all times [46]. This is the core element of the hypothesized effectiveness of the intervention. The placebo group will use a non-adaptive (fixed low-level training) version of the training program. The interface of both the placebo and the training version is identical, but the difficulty level in the placebo version does not increase adaptively as the patient performance improves. The study involves three examinations at the following time points: baseline before training, retesting about 1 month after training completion, and follow-up retesting four months after training completion in order to examine the longer-term effects, see Table 2 for details.

\section{Randomization}

Participants in the study are randomly allocated to either the adaptive training group or the non-adaptive (placebo) training group after the baseline assessment. The patients randomized to the placebo group will be offered the adaptive training after the 4-month follow-up examination. The neuropsychologist and radiologist carrying out the assessments are blinded to group adherence.

Both the adaptive and the placebo training groups undergo a 25-session standardized computerized WM training within the same time period (all participants start training/placebo at the same time), differing only in the adaptability of the training. During the training phase, weekly follow-ups by standardized phone interviews are scheduled for all participants regardless of group affiliation. After the initial training phase, the participants are examined at 1 and 4 months after finalizing their training. All participant contact is logged in order to discover potential performance bias. In addition to the research team, the patients are followed by their primary physician, who will tend to all non-trial-related issues.

\section{Sample size and power calculation}

Using pre-training and post-training test scores obtained by Brehmer et al. [47], 72 patients are needed for inclusion in the study to obtain a statistical power of $80 \%$. Allowing for a maximum dropout rate of $20 \%$, the number of study subjects needed for inclusion has been set to 90 patients with MCI. Power calculations are based on the primary outcome measure, which is change in WM capacity assessed by neuropsychological tests and behavioral inventories that assess WM and related functions. The statistical power measures will be calculated using Sample Power 2.0.

Table 2 Assessment measures at baseline and post-intervention evaluations

\begin{tabular}{|c|c|c|c|}
\hline \multirow[t]{2}{*}{ Assessment tool } & \multirow{2}{*}{$\begin{array}{c}\text { Baseline } \\
\text { evaluation }\end{array}$} & \multicolumn{2}{|c|}{ Post-intervention evaluation } \\
\hline & & $\begin{array}{c}\text { 1: } 1 \text { month } \\
\text { after training }\end{array}$ & $\begin{array}{l}\text { 2: } 4 \text { months } \\
\text { after training }\end{array}$ \\
\hline Medical examination & $x$ & & \\
\hline MR caput & $x$ & $x$ & $x$ \\
\hline Spinal fluid & $x$ & & \\
\hline DNA sampling & $x$ & & \\
\hline WAIS-IV & $x$ & & \\
\hline WMS-III & $x$ & $x$ & $x$ \\
\hline Digit span & $x$ & $x$ & $x$ \\
\hline Spatial span & $x$ & $x$ & $x$ \\
\hline Letter-Number Sequencing & $x$ & $x$ & $x$ \\
\hline CVLT-II & $x$ & $x$ & $x$ \\
\hline D-KEFS & $x$ & $x$ & $x$ \\
\hline IGT & $x$ & & \\
\hline Vineland & $x$ & & $x$ \\
\hline BRIEF-A & $x$ & $x$ & $x$ \\
\hline DEX & $x$ & $x$ & $x$ \\
\hline
\end{tabular}




\section{Statistical analysis}

Performance gain in the assessment scores related to WM function from baseline is the primary outcome of interest. Participants' daily performance on the WM tasks within the program will be aggregated into one $t$-standardized performance score. Weekly performance scores will be used for analysis. A repeated-measure ANOVA will be conducted to investigate performance gain during the training period. One-way ANOVA will be conducted separately for the assessment criterion and transfer tasks and the rating scales of functioning, to examine potential baseline differences between the intervention and the placebo groups. To determine differences in training-related changes, a general linear mixed model with appropriate covariates will be conducted (baseline, post-training, and follow-up), and within-subject factors will be used for the cognitive tasks and assessment data. For all analyses, $\alpha$ levels will be set to 0.05 and effect sizes refer to partial $\eta$-square values. With regard to cerebral MRI, the DTI images will be analyzed using the software FSL with [48] to provide information on any changes in white matter microstructure with training. Automated morphometry to evaluate cortical thickness and surface area, total gray and white matter volumes, and deep nuclei volumes will be performed using the freely available FreeSurfer software program, v5.1 [49]. Complete-case analysis will be used where missing data are excluded from the analysis. A fully detailed statistical analysis will be performed before unmasking of the data.

\section{Dissemination plan}

The dissemination plan includes five scientific papers that will be submitted to peer-reviewed medical journals:

1. Computer-based adaptive working memory training on cognitive functioning in elderly patients with mild cognitive impairment assessed by neuropsychological tests. A randomized double-blind controlled study.

2. Does cognitive training in patients with Mild Cognitive Impairment relate to structural changes in the white and gray matter of the brain?

3. Do patients with MCI with a particular genotype for the $L M X 1 A$ gene (i.e, AA alleles) or for APOE (i.e. non- $\varepsilon 4)$ have stronger training effects after adaptive working memory training?

4. Does computer -based working memory training have secondary effects on executive function and activities of daily living in elderly patients with MCI? A randomized, double-blind controlled study.

5. Long-term follow-up of elderly patients with mild cognitive impairment after completion of an adaptive, computerized, standardized, and intensive working memory program: neuropsychological and daily-life functioning.

The first publication will be submitted at the end of 2014, and the other articles will be submitted before the project ends in 2017. In addition, the results will be disseminated at international conferences and national forums, and in the popular press to target a wide range of groups, including researchers, healthcare providers, and health-care professionals, as well as the general public. The results will be disseminated regardless of the magnitude or direction of effect.

\section{Discussion}

At present, there is no known cure for memory decline in the elderly or other cognitive deficits that follows MCI. Discovery of a method capable of enhancing memory, or postponing further decline in WM function in patients in the early stages of dementia, would be of great importance for a large and growing patient group.

The strengths of this trial are the extensive assessment of the participants on several levels, including gene samples, quantitative brain imaging, and neuropsychological testing. We hope the assessment will provide new and important information on the memory abilities and daily life functioning in elderly patients with MCI. This extensive assessment is relevant because of the complex nature of the etiology and behavioral outcomes of memory decline in elderly patients, and the heterogeneity of the cognitive phenotypes seen in memory clinics. The proposed study will be the first to investigate the possible mediating influence of biomarkers on the effects of cognitive training in patients with MCI. Such biomarkers may contribute in prediction of training effects. Furthermore, identification of a genetic marker to assess WM trainability would be an important finding that would allow cognitive intervention to be offered to those best able to benefit from it. This would also be of benefit to society.

Participating in this study involves no negative effects for the patients; but it may help the patients to improve their everyday functioning, and perhaps also to postpone further decline in WM function. The study also seeks to identify cognitive reserves in these patients, as such reserves seem to play an important role in the development of cognitive impairment [50]. Currently, there is a lack of knowledge of the effects of adaptive, standardized and intensive computerized WM training on individuals with $\mathrm{MCI}$, and this study will be able to provide new and unique knowledge. If WM training can be shown to have positive effects on cognitive and daily-life functioning in people with $\mathrm{MCI}$, a new approach to treatment of memory decline may be feasible. 


\section{Trial status}

The trial started in August 2013, and is currently enrolling participants.

\section{Abbreviations}

BRIEF-A: Brief Rating Inventory of Executive Functions Adult version;

CVLT-II: California Verbal Learning Test, second edition; D-KEFS: Delis-Kaplan Executive Function System; DEX: Behavioral assessment of the Dysexecutive Syndrome Questionnaire; IGT: lowa Gambling Task; MCl: Mild Cognitive Impairment; Vineland: The Vineland Adaptive Behavior Scales, second edition (interview edition); WAIS-IV: Wechsler Adult Intelligence Scale, fourth edition; WMS-III: Wechsler Memory Scale, third edition.

\section{Competing interests}

The authors declare that they have no competing interests.

\section{Authors' contributions}

MMF: conception and design, data collection and analysis, manuscript writing, and final approval of the manuscript. SSH: conception and design, data collection and analysis, manuscript writing, and final approval of the manuscript. JS: conception and design, data interpretation, manuscript writing, critical revision, and final approval of the manuscript. GCCL: conception and design, manuscript writing, critical revision, and final approval of the manuscript. All authors read and approved the final manuscript.

\section{Acknowledgements}

We are grateful for the funding from the South-Eastern Norway Regional Health Authority and Sorlandet Hospital HF.

\section{Author details}

'Department of Medicine, Geriatric Unity, The Memory Clinic, Sørlandet Hospital, Arendal, Norway. ${ }^{2}$ Department of Pediatrics, Sørlandet Hospital, Arendal, Norway. ${ }^{3}$ Department of Laboratory Medicine, Children's and Women's Health, Norwegian University of Science and Technology, Trondheim, Norway.

Received: 17 February 2014 Accepted: 8 April 2014

Published: 3 May 2014

\section{References}

1. Petersen RC, Negash S: Mild cognitive impairment: an overview. CNS Spectr 2008, 13(1):45-53.

2. Petersen RC, Smith GE, Waring SC, Ivnik RJ, Tangalos EG, Kokmen E: Mild cognitive impairment: clinical characterization and outcome. Arch Neurol 1999, 56(3):303-308.

3. Saunders NL, Summers MJ: Attention and working memory deficits in mild cognitive impairment. J Clin Exp Neuropsychol 2010, 32(4):350-357.

4. Baddeley A: Working memory: looking back and looking forward. Nat ReV Neurosci 2003, 4(10):829-839.

5. Baddeley AD, Hitch GLL: Working memory. In The psychology of learning and motivation: Advances in research and theory, Volume 8. Edited by Bower GA. New York: Academic; 1974:47-89.

6. Fry AF, Hale S: Relationships among processing speed, working memory, and fluid intelligence in children. Biol Psychol 2000, 54(1-3):1-34.

7. Salthouse TA: Mental exercise and mental aging: evaluating the validity of the "Use it or lose it" hypothesis. Perspect Psycho/ Sci 2006, 1:68-87.

8. Sitzer DI, Twamley EW, Jeste DV: Cognitive training in Alzheimer's disease: a meta-analysis of the literature. Acta Psychiatr Scand 2006, 114(2):75-90.

9. Jolles DD, Crone EA: Training the developing brain: a neurocognitive perspective. Front Hum Neurosci 2012, 6:76.

10. Klingberg T: Training and plasticity of working memory. Trends Cogn Sci 2010, 14(7):317-324.

11. Klingberg T: Development of a superior frontal-intraparietal network for visuo-spatial working memory. Neuropsychologia 2006, 44(11):2171-2177.

12. Engvig A, Fjell AM, Westlye ET, Moberget $T$, Sundseth $\varnothing$, Larsen VA, Walhovd KB: Effects of memory training on cortical thickness in the elderly. Neuroimage 2010, 52(4):1667-1676.

13. Takeuchi H, Sekiguchi A, Taki Y, Yokoyama S, Yomogida Y, Komuro N, Yamanouchi T, Suzuki S, Kawashima R: Training of working memory impacts structural connectivity. J Neurosci 2010, 30(9):3297-3303.
14. Takeuchi H, Taki Y, Hashizume H, Sassa Y, Nagase T, Nouchi R, Kawashima R: Effects of training of processing speed on neural systems. J Neurosci 2011, 31(34):12139-12148.

15. Johansson B, Tornmalm M: Working memory training for patients with acquired brain injury: effects in daily life. Scand J Occup Ther 2012, 19(2):176-183

16. Stott J, Spector A: A review of the effectiveness of memory interventions in mild cognitive impairment (MCl). Int Psychogeriatr 2011, 23(4):526-538.

17. Thorell LB, Lindqvist S, Bergman Nutley S, Bohlin G, Klingberg T: Training and transfer effects of executive functions in preschool children. Dev Sci 2009, 12(1):106-113.

18. Bergman Nutley S, Söderqvist S, Bryde S, Thorell LB, Humphreys K, Klingberg T: Gains in fluid intelligence after training non-verbal reasoning in 4-year-old children: a controlled, randomized study. Dev Sci 2011, 14(3):591-601.

19. Gibson BS, Gondoli DM, Johnson AC, Steeger CM, Dobrzenski BA, Morrissey RA: Component analysis of verbal versus spatial working memory training in adolescents with ADHD: a randomized, controlled trial. Child Neuropsychol 2011, 17(6):546-563.

20. Roughan $L$, Hadwin JA: The impact of working memory training in young people with social, emotional and behavioural difficulties. Learn Individ Differ 2011, 21(6):759-764.

21. Diamond A, Lee $\mathrm{K}$ : Interventions shown to aid executive function development in children 4 to 12 years old. Science 2011, 333(6045):959-964.

22. Løhaugen GCC, Antonsen I, Håberg A, Gramstad A, Vik T, Brubakk AM, Skranes J: Computerized working memory training improves function in adolescents born at extremely low birth weight. J Pediatr 2011, 158(4):555-U56.

23. Løhaugen GCC, Gramstad A, Evensen KA, Martinussen M, Lindqvist S, Indredavik M, Vik T, Brubakk AM, Skranes J: Cognitive profile in young adults born preterm at very low birthweight. Dev Med Child Neurol 2010, 52(12):1133-1138.

24. Dahlin KIE: Effects of working memory training on reading in children with special needs. Read Writ 2011, 24(4):479-491.

25. Holmes J, Place M, Dunning DL, Hilton KA, Elliott JG: Working memory deficits can be overcome: impacts of training and medication on working memory in children with ADHD. Appl Cogn Psychol 2010, 24(6):827-836.

26. Kronenberger WG, Pisoni DB, Henning SC, Colson BG, Hazzard LM: Working memory training for children with cochlear implants: a pilot study. J Speech Lang Hearing Res 2011, 54(4):1182-1196.

27. Lundqvist A, Grundström K, Samuelsson K, Rönnberg J: Computerized training of working memory in a group of patients suffering from acquired brain injury. Brain Inj 2010, 24(10):1173-1183.

28. Mezzacappa E, Buckner JC: Working memory training for children with attention problems or hyperactivity: a school-based pilot study. Sch Ment Heal 2010, 2(8):202-208.

29. Klingberg $T$, Forssberg $H$, Westerberg $H$ : Training of working memory in children with ADHD. J Clin Exp Neuropsychol 2002, 24(6):781-791.

30. Olesen PJ, Westerberg $H$, Klingberg T: Increased prefrontal and parietal activity after training of working memory. Nat Neurosci 2004, 7(1):75-79.

31. Westerberg $H$, Klingberg T: Changes in cortical activity after training of working memory-a single-subject analysis. Physiol Behav 2007, 92(1-2):186-192.

32. Westerberg $H$, Jacobaeus $H$, Hirvikoski T, Clevberger $P$, Ostensson ML, Bartfai A, Klingberg T: Computerized working memory training after stroke-a pilot study. Brain Inj 2007, 21(1):21-29.

33. Gates J, Sachdev PS, Fiatarone Singh MA, Valenzuela M: Cognitive and memory training in adults at risk of dementia: a systematic review. BMC Geriatr 2011, 11:55.

34. Li H, Li J, Li N, Li B, Wang P, Zhou T: Cognitive intervention for persons with mild cognitive impairment: a meta-analysis. Ageing Res Rev 2011, 10(2):285-296

35. Bellander $\mathrm{M}$, Brehmer $\mathrm{Y}$, Westerberg $\mathrm{H}$, Karlsson S, Fürth D, Bergman $\mathrm{O}$ Eriksson E, Bäckman L: Preliminary evidence that allelic variation in the LMX1A gene influences training-related working memory improvement. Neuropsychologia 2011, 49(7):1938-1942.

36. Soderqvist S, Bergman Nutley S, Peyrard-Janvid M, Matsson H, Humphreys K, Kere J, Klingberg T: Dopamine, working memory, and training induced plasticity: implications for developmental research. Dev Psychol 2012, 48(3):836-843. 
37. Bäckman L, Nyberg L, Soveri A, Johansson J, Andersson M, Dahlin E, Neely AS, Virta J, Laine M, Rinne JO: Effects of working-memory training on striatal dopamine release. Science 2011, 333(6043):784.

38. McNab F, Varrone A, Farde L, Jucaite A, Bystritsky P, Forssberg H, Klingberg $\mathrm{T}$ : Changes in cortical dopamine D1 receptor binding associated with cognitive training. Science 2009, 323(5915):800-802.

39. Brehmer $Y$, Westerberg $H$, Bellander $M$, Fürth $D$, Karlsson S, Bäckman L: Working memory plasticity modulated by dopamine transporter genotype. Neurosci Lett 2009, 467(2):117-120.

40. Durstewitz D, Seamans JK: The computational role of dopamine D1 receptors in working memory. Neural Netw 2002, 15(4-6):561-572.

41. Schulz KF, Altman DG, Moher D, CONSORT Group: CONSORT 2010 statement: updated guidelines for reporting parallel group randomised trials. Int I Surg 2011, 9(8):672-677.

42. Boutron I, Moher D, Altman DG, Schulz K, Ravaud P, for the CONSORT group: Extending the CONSORT statement to randomized trials of nonpharmacologic treatment: explanation and elaboration. Ann Intern Med 2008, 148:W-60-W-66.

43. D'Esposito M, Postle BR, Rypma B: Prefrontal cortical contributions to working memory: evidence from event-related fMRI studies. Exp Brain Res 2000, 133(1):3-11.

44. Rabin LA, Paré N, Saykin AJ, Brown MJ, Wishart HA, Flashman LA, Santulli RB: Differential memory test sensitivity for diagnosing amnestic mild cognitive impairment and predicting conversion to Alzheimer's disease. Neuropsychol Dev Cogn B Aging Neuropsychol Cogn 2009, 16(3):357-376.

45. Lezak MD, Howieson DB, Loring DW: Neuropsychological assessment. New York: Oxford University Press; 2004.

46. Klingberg T, Fernell E, Olesen PJ, Johnson M, Gustafsson P, Dahlström K, Gillberg CG, Forssberg H, Westerberg H: Computerized training of working memory in children with ADHD-a randomized, controlled trial. J Am Acad Child Adolesc Psychiatry 2005, 44(2):177-186.

47. Brehmer $Y$, Westerberg $H$, Backman L: Working-memory training in younger and older adults: training gains, transfer, and maintenance. Front Hum Neurosci 2012, 6:63.

48. University_of_Oxford. FMRIB Software Library, Release 4.1: University_of_Oxford. FMRIB Software Librany, Release 4.1; 2008. [cited 2012 01.09]; Available from: http://www.fmrib.ox.ac.uk/fsldownloads/.

49. Biomedical_Imaging, A.A.M.C: Freesurfer image analysis suite (cited 01.05 2014). Available from: http://surfer.nmr.mgh.harvard.edu.

50. Scarmeas N, Stern Y: Cognitive reserve: implications for diagnosis and prevention of Alzheimer's disease. Curr Neurol Neurosci Rep 2004, 4(5):374-380.

doi:10.1186/1745-6215-15-156

Cite this article as: Flak et al:: The Memory Aid study: protocol for a randomized controlled clinical trial evaluating the effect of computer-based working memory training in elderly patients with mild cognitive impairment (MCl). Trials 2014 15:156.

\section{Submit your next manuscript to BioMed Central and take full advantage of:}

- Convenient online submission

- Thorough peer review

- No space constraints or color figure charges

- Immediate publication on acceptance

- Inclusion in PubMed, CAS, Scopus and Google Scholar

- Research which is freely available for redistribution 